\title{
Relationship between Nonadiabaticity and Damping in Permalloy Studied by Current Induced Spin Structure Transformations
}

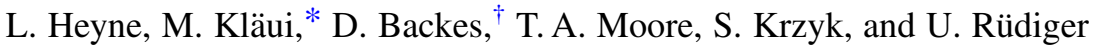 \\ Fachbereich Physik, Universität Konstanz, Universitätsstrasse 10, D-78457 Konstanz, Germany \\ L. J. Heyderman \\ Laboratory for Micro- and Nanotechnology, Paul Scherrer Institut, CH-5232 Villigen PSI, Switzerland \\ A. Fraile Rodríguez and F. Nolting \\ Swiss Light Source, Paul Scherrer Institut, CH-5232 Villigen PSI, Switzerland
}

T. O. Mentes, M. Á. Niño, and A. Locatelli

Sincrotrone Trieste S.C.p.A., 34012 Basovizza, Trieste, Italy

K. Kirsch and R. Mattheis

Institute of Photonic Technology (IPHT), Jena, P.O. Box 100239, D-07702 Jena, Germany

\begin{abstract}
By direct imaging we determine spin structure changes in Permalloy wires and disks due to spin transfer torque as well as the critical current densities for different domain wall types. Periodic domain wall transformations from transverse to vortex walls and vice versa are observed, and the transformation mechanism occurs by vortex core displacement perpendicular to the wire. The results imply that the nonadiabaticity parameter $\beta$ does not equal the damping $\alpha$, in agreement with recent theoretical predictions. The vortex core motion perpendicular to the current is further studied in disks revealing that the displacement in opposite directions can be attributed to different polarities of the vortex core.
\end{abstract}

PACS numbers: 72.25.Ba, 75.60.Ch, 75.75.+a

Controlled manipulation of magnetic systems by current injection has become an exciting field of research since its theoretical prediction more than ten years ago $[1,2]$. Recently the feasibility of current-induced domain wall motion (CIDM) has been demonstrated by a number of groups using different techniques [3-8].

In a magnetic wire, two head-to-head domain wall (DW) configurations prevail: the vortex wall (VW) [spin structure in Figs. 1(a) and 1(b)] and the transverse wall (TW) [see Figs. 1(c) and 1(d)]. The TW is similar to a Néel wall, where the magnetization rotates in-plane by $180^{\circ}$. In a VW the magnetization curls around a vortex core, where the magnetization points out-of-plane to reduce the exchange energy. The VW can be characterized by the sense of rotation of the in-plane magnetization [e.g., clockwise in Fig. 1(a)] and the polarity of the vortex core, that is to say the direction of the out-of-plane magnetic component (up or down). Injecting a spin-polarized current across the DW results in a displacement of the DW in the direction of the electron flow, which for VWs has been observed experimentally by a number of groups [5-7]. While TWs have been obtained as a result of a single transformation of a VW to a TW, these TWs could not be moved anymore [7], so that to date no direct imaging of the displacement or the transformations of TWs has been made available. Furthermore the physical reason for the transformation could not be unambiguously determined, since only one single transformation from a VW to a TW was observed.
In order to address the spin torque effect theoretically, additional terms have been added to the Landau-LifshitzGilbert (LLG) equation [9-13]. These spin torque terms corresponding to the influence of the spin-polarized current are shown in the explicit form in the following equation for the current flowing in the $x$ direction [1215]: $\tau=-\frac{u_{x}}{1+\alpha^{2}}\left[(1+\beta \alpha) \frac{\partial \mathbf{S}}{\partial x}+(\alpha-\beta) \mathbf{S} \times \frac{\partial \mathbf{S}}{\partial x}\right]$.

$\tau$ represents the current-induced torque acting on the (dimensionless) spin $\mathbf{S}$. $u_{x}$ is given by $u_{x}=$ $j_{e} P g \mu_{B} /\left(2 e M_{s}\right)$ with current density $j_{e}$, polarization $P$, and the saturation magnetization $M_{S}$ [14]. $\alpha$ is the damping constant and $\beta$ the nonadiabaticity parameter. While these extra terms explain CIDM, they also predict a distortion of the DW depending on the choice of parameters [11]. In particular, the last term is responsible for an out-of-plane torque, which leads to a deformation of the DW spin structure resulting in DW transformations.

In a certain range of wire dimensions both DW types can coexist since they both constitute local energy minima [16] and periodic transformations are predicted in these wires under current injection [12]. Starting with a clockwise VW [see Fig. 1(a)], the vortex core will feel a force perpendicular to the current so that it is moved not only in the electron flow direction but also perpendicularly towards the wire edge. The direction of this perpendicular displacement is determined by the polarity of the vortex core and the sign of $(\alpha-\beta)$. For large enough current densities the vortex core is expelled and a TW is formed [see Fig. 1(c), 

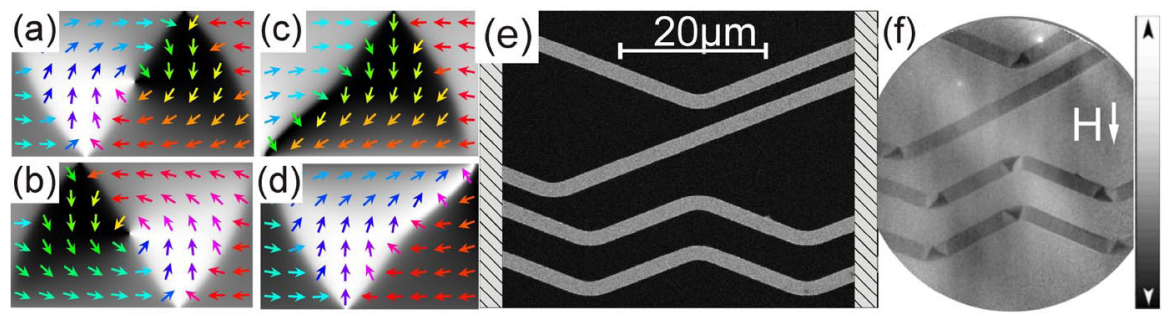

FIG. 1 (color online). (a),(b) Simulations of a clockwise and a counterclockwise VW, respectively. (c),(d) TWs with opposite transverse components of the wall spin structure [25]. (e) SEM image of the wavy lines (1500 nm wide, $8 \mathrm{~nm}$ thick). The Au pads on either end are shaded. (f) XMCD image showing the magnetization configuration after applying an external field $\mathrm{H}$ along the direction indicated by the arrow. TWs are formed at the kinks. Different shades of gray indicate the orientation of the magnetic spins (see gray scale bar).

assuming the vortex core moved down). Then a new vortex is nucleated with the same clockwise sense of rotation as in Fig. 1(a) but with opposite polarity, which starts to move in the opposite direction (upwards). So a new VW is formed with the same in-plane contrast as the former one but an opposite out-of-plane component. When the vortex core is expelled at the upper edge, a TW is created [see Fig. 1(d)] with the opposite transverse component of the wall spin structure as the previous TW. Then again a new vortex core is nucleated and this periodic transformation process continues as long as current is injected. During this whole process the in-plane sense of rotation of the VWs stays the same as the original one, and a counterclockwise VW as shown in Fig. 1(b) does not occur.

The details of the transformations are governed by the nonadiabaticity parameter $\beta$, and a number of theoretical models exist to calculate $\beta$. Zhang and $\mathrm{Li}$ [15] argued that $\beta$ should be in the range of $10^{-2}$. They further found that this factor is crucial for continuous CIDM. Xiao et al. [17] cast doubt on the existence of the nonadiabaticity parameter due to spin-flip scattering but introduce a spatially varying $\beta$ that depends on the gradient of the magnetization.

The most hotly debated issue is the relation between $\beta$ and the damping constant $\alpha$, since this also depends on the type of damping used (Landau-Lifshitz damping vs Gilbert damping) [18]. Kohno et al. [19] calculated microscopically the nonadiabaticity parameter from the spin relaxation of conduction electrons and found that in general $\beta \neq \alpha$. In contrast, Tserkovnyak et al. [20] and Barnes et al. [21] claim that $\beta$ should equal $\alpha$. This view has become very popular since it allows one to use the LandauLifshitz damping rather than the Gilbert type of damping, which some consider to be the more appropriate type [18,22].

Under the assumption that $\beta=\alpha$, no transverse movement of the vortex core and thus no transformations should occur in a wire. Up to the present time no reliable experimental data have become available and experiments addressing this topic are thus of utmost importance.

In this Letter we investigate current-induced DW motion and transformations in ferromagnetic Permalloy (Py) zigzag lines. We determine the transformations and vortex core displacements for consecutive pulse injections to in- vestigate whether random thermal excitations or spin transfer torque effects govern the spin structure changes and gain insights into the nature of $\beta$. The critical current densities for VWs and TWs are determined and compared.

In Py disks, vortex core displacement is observed in the direction perpendicular to the current, which is correlated with the vortex core polarity.

Figure 1(e) shows a scanning electron microscopy (SEM) image of the Py wavy lines that are $8 \mathrm{~nm}$ thick and $1500 \mathrm{~nm}$ wide. The lines are contacted by Au pads on both ends for current injection. The samples were produced on a Si substrate by electron beam lithography and a twostep lift-off process [23].

$\mathrm{X}$-ray magnetic circular dichroism (XMCD) photoemission electron microscopy is used to image the magnetization [24] with an angle of incidence for the $\mathrm{x}$-ray beam of $16^{\circ}$. An XMCD image of the initial configuration is presented in Fig. 1(f), where the wires were magnetized inplane with an external field along the direction indicated by the arrow. After reducing the field, transverse DWs are formed at the kinks. The wire geometry was chosen to be close to the phase boundary between VWs and TWs so that both kinds of DWs are stable [16].

After the initialization, we inject $25 \mu$ s long unipolar current pulses with a current density of about $1 \times$ $10^{12} \mathrm{~A} / \mathrm{m}^{2}$. The magnetization is imaged after each injected pulse at room temperature. We observe that current injections displace the DWs in the electron flow direction and also induce DW transformations. After the first current injection, transformations of the initial TWs to VWs are observed. Subsequent current injections result in transformations back to TWs. Further injections can then again transform the TWs to VWs.

Different reasons could be responsible for such transformations: spin transfer torque is predicted to yield such transformations for $\beta \neq \alpha$, but transformations can also be induced by thermal activation and defects [16]. To distinguish between the causes we can make use of the fact that for a given initial DW configuration the spin transfer torque predicts transformations that result always in the same sense of rotation for the VWs [12,25]. If the observed transformations were thermally or defect induced, both senses of rotation should occur equally often. From 17 
observed transformations from a TW we only saw transformations to a clockwise VW, and no counterclockwise VW occurred. The DWs were located on different wires and kinks, but all had the same initial configuration [see DW in Fig. 2(a)]. This symmetry breaking feature clearly rules out thermal activation and defects, but perfectly fits to the spin torque model under the assumption that $\alpha \neq \beta$, which is in agreement with the theoretical prediction of Kohno et al., for instance [19]. Furthermore, for more than 30 observed current-induced VW motions the sense of rotation stayed constant and did not change, as expected by theory.

An example of a sequence of observed transformations for one DW is presented in Fig. 2. The top row shows the recorded images after subsequent current injections, and the bottom row shows results from a corresponding micromagnetic simulation [25]. The initial TW located at a kink [Fig. 2(a)] is displaced after the first current injection and additionally transformed into a clockwise VW [Fig. 2(b)]. A second injection again displaces the DW and results in a TW with an opposite transverse component of the wall spin structure compared to the first [Fig. 2(c)]. The next injection yields an off-center VW [Fig. 2(d)] and the wall then attains again a clockwise VW structure after another injection [Fig. 2(e)]. Note that the different contrast of the VWs in Figs. 2(b) and 2(e) arises from the fact that they were located on different branches of the zigzag wire, which is taken into account in the corresponding simulations.

The transformation mechanism due to the spin torque model is based on the displacement of the vortex core perpendicular to the current towards the wire edge. We obtain direct evidence for this mechanism by the observation of an off-center VW as shown in Fig. 2(d). Normally this state should immediately relax back into a VW, but probably a defect stabilizes the vortex core in this offcenter position. This intermediate state occurs during the transformation process and is direct evidence for the perpendicular motion and eventual annihilation of the vortex core predicted by theory.

In this geometry we can carry out another important experiment to understand the previous results about the DW that could not be moved anymore after it had trans- formed from a VW to a TW [7]. Since we observe VWs and TWs in the same geometry, their critical current densities can be directly compared. The lowest critical current density at which CIDM was observed for TWs is $9 \times$ $10^{11} \mathrm{~A} / \mathrm{m}^{2}$, whereas CIDM for VWs was observed already at $7.5 \times 10^{11} \mathrm{~A} / \mathrm{m}^{2}$. According to recent calculations [13], this can be explained by a stronger pinning for TWs at edge irregularities and explains why the TW in [7] could not be moved with the same current densities as the VW from which it transformed.

Current-induced vortex core displacement is predicted also to occur in magnetic disks, and thus we have studied current injection into Py disks containing a vortex spin structure. An SEM image of a disk is presented in Fig. 3(a). A vortex core is formed in the center of the structure as visible in the XMCD image shown in Fig. 3(b).

Current injection will displace the vortex perpendicular to the current until the spin torque is compensated by a restoring force that tries to move the vortex back into the center position. The direction of the displacement is predicted to depend on the vortex core polarity [26]. After switching off the current, the vortex will relax back to the center, but defects can pin the vortex core and keep it at the off-center position even after the current is switched off.

To investigate the current-induced vortex core displacement we injected current pulses with alternating direction into the Py disks. The vortex core polarity was defined prior to injection by a strong external out-of-plane magnetic field. We observe that if in the same disk the vortex core polarity has been changed, the current direction also had to be reversed to obtain a displacement in the same direction. This behavior cannot be explained by the Oersted field generated by the current, since an Oersted field will displace the vortex core parallel to the current and depends mainly on the in-plane sense of rotation, which stayed constant. From this we can conclude that the vortex core displacement is a result of the spin transfer torque and the direction of the displacement depends on the polarity of the vortex core.

Displacements for one polarity are shown in Fig. 3. After a current pulse $\left(6.5 \times 10^{11} \mathrm{~A} / \mathrm{m}^{2}, 25 \mu \mathrm{s}\right.$ long $)$, the cen-
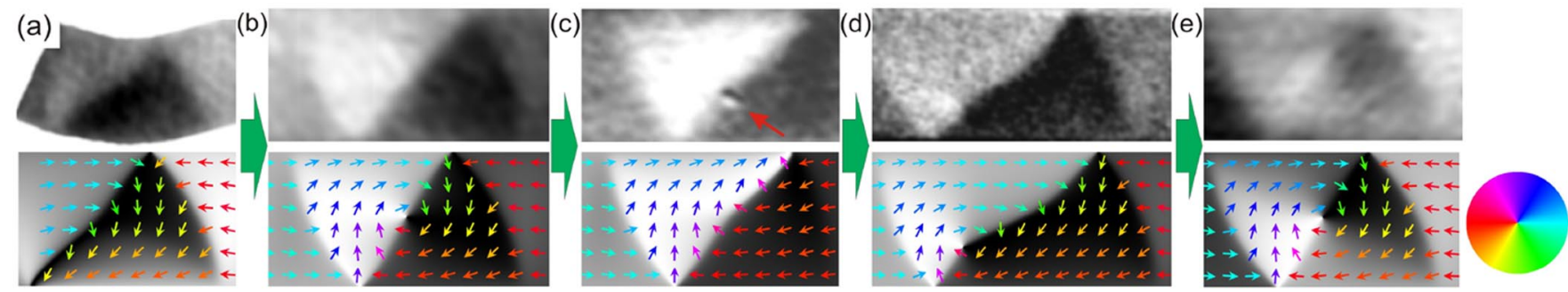

FIG. 2 (color online). XMCD images of current-induced DW transformations. The bottom row shows results from a corresponding simulation [25] with the contrast direction equivalent to the XMCD images. The arrow color code is indicated by the color disk. The direction of contrast is horizontal $\pm 20^{\circ}$ depending on the location on the curved wire. (a) Initial TW at the kink. (b) A $25 \mu$ s pulse transforms the TW to a clockwise VW. (c) Further injection results in a TW with the opposite transverse component of the wall spin structure compared to the first. The marked dark spot originates from a channel plate defect in the imaging unit. (d) The next injection yields an off-center VW. (e) Subsequent injection results again in a VW with the same sense of rotation as in (b) and (d). 


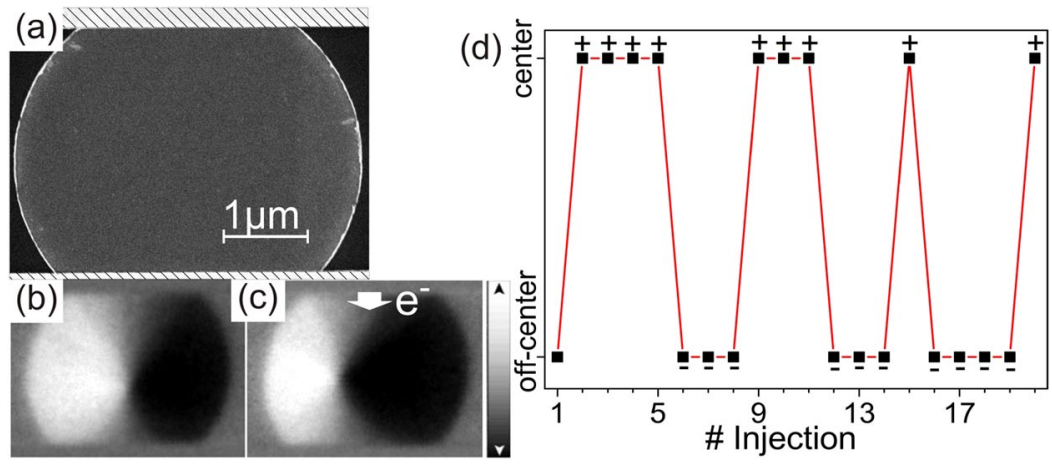

FIG. 3 (color online). (a) SEM image of the Py disk ( $4 \mu \mathrm{m}$ wide, $20 \mathrm{~nm}$ thick). The $\mathrm{Au}$ contacts are shaded. (b) The corresponding magnetization configuration. The gray scale bar indicates the magnetic orientation. (c) Off-center magnetization after single pulse injection $\left(6.5 \times 10^{11} \mathrm{~A} / \mathrm{m}^{2}, 25 \mu \mathrm{s}\right.$ long). (d) Position of the vortex core vs consecutive injected pulses, the sign at the data points indicates whether a positive or negative pulse has been injected.

tered vortex is shifted about $500 \mathrm{~nm}$ perpendicular to the current flow and pinned at an off-center position [Fig. 3(c)]. A second pulse with reversed direction brings back the original centered vortex core [see Fig. 3(b)]. This is repeated several times with varying current direction and the result is shown in Fig. 3(d). The relative position of the vortex core perpendicular to the current is shown as a function of the injected pulses. Negative pulses always shift the vortex off-center to the left, whereas positive pulses result again in a centered vortex. The fact that we observe a shift in only one direction indicates that there is just one pinning site close to the structure center strong enough to stabilize the off-center vortex. The experiment was also conducted on other disks where we observed the same kind of displacement with one pinning center. In a few disks there was no permanent displacement; presumably no defects are present and so the vortex core always relaxes back into the center.

Using the theoretical predictions from Ref. [26], a final equilibrium core displacement during the pulse of about $200 \mathrm{~nm}$ is expected for our setup. However, the vortex will conduct a precessional motion and the maximum distance from the equilibrium position will be about twice the equilibrium distance. This is in the range of the distance from the center to the defect. Thus the spin torque is able to drag the vortex to the defect, in agreement with our observation that for lower current densities the permanent shift of the vortex to the defect after pulse injection becomes less likely.

In conclusion, we have given direct evidence for the vortex core displacement due to the spin transfer torque in magnetic wires as well as in magnetic disks. The observed DW transformations in the $1500 \mathrm{~nm}$ wide and $8 \mathrm{~nm}$ thick Py wires are in very good agreement with theory of the spin transfer torque, while thermal activation and defects can be ruled out as a cause. Moreover, the transformations can only be reproduced theoretically for $\beta \neq \alpha$. Comparing the critical current densities $j_{c}$ for TWs and VWs we find a larger $j_{c}$ for TWs in agreement with theory, which explains the surprising stopping of TWs in earlier experiments. Direct observation of vortex core displacement perpendicular to the current was observed in Py disks, and the displacement in opposite directions is attributed to the different polarities of the vortex cores.
The authors acknowledge support by the DFG (No. SFB 513), by the EU through the European Regional Development Fund (Interreg III A Program), the Human Resources \& Mobility programme (Marie Curie Actions), the Samsung Advanced Institute of Technology, and the Landesstiftung Baden-Württemberg. Part of this work was performed at the Swiss Light Source, Paul Scherrer Institut Villigen, Switzerland (Fig. 3) and at ELETTRA, Trieste, Italy [Figs. 1(f) and 2].

*mathias.klaeui@uni-konstanz.de

${ }^{\dagger}$ Also at LMN, Paul Scherrer Institut, CH-5232 Villigen, Switzerland.

[1] L. Berger, Phys. Rev. B 54, 9353 (1996).

[2] J. C. Slonczewski, J. Magn. Magn. Mater. 159, L1 (1996).

[3] N. Vernier et al., Europhys. Lett. 65, 526 (2004).

[4] J. Grollier et al., Appl. Phys. Lett. 83, 509 (2003).

[5] A. Yamaguchi et al., Phys. Rev. Lett. 92, 077205 (2004).

[6] L. Thomas et al., Nature (London) 443, 197 (2006).

[7] M. Kläui et al., Phys. Rev. Lett. 95, 026601 (2005).

[8] M. Kläui et al., Appl. Phys. Lett. 83, 105 (2003); Phys. Rev. Lett. 94, 106601 (2005).

[9] Z. Li and S. Zhang, Phys. Rev. B 70, 024417 (2004).

[10] Z. Li and S. Zhang, Phys. Rev. Lett. 92, 207203 (2004).

[11] J. He et al., Phys. Rev. B 73, 184408 (2006).

[12] A. Thiaville et al., Europhys. Lett. 69, 990 (2005).

[13] J. He et al., J. Appl. Phys. 99, 08G509 (2006).

[14] C. Schieback et al., Eur. Phys. J. B 59, 429 (2007).

[15] S. Zhang and Z. Li, Phys. Rev. Lett. 93, 127204 (2004).

[16] M. Laufenberg et al., Appl. Phys. Lett. 88, 052507 (2006).

[17] J. Xiao et al., Phys. Rev. B 73, 054428 (2006).

[18] W. M. Saslow et al., APS March Meeting 2007-J14.7 http://meetings.aps.org/link/BAPS.2007.MAR.J14.7.

[19] H. Kohno et al., J. Phys. Soc. Jpn. 75, 113706 (2006).

[20] Y. Tserkovnyak et al., Phys. Rev. B 74, 144405 (2006).

[21] S. E. Barnes et al., Phys. Rev. Lett. 95, 107204 (2005).

[22] M. D. Stiles et al., Phys. Rev. B 75, 214423 (2007).

[23] L. J. Heyderman et al., Microelectron. Eng. 73-74, 780 (2004).

[24] J. Stöhr et al., Science 259, 658 (1993).

[25] The simulation was carried out using LLG (http:// llgmicro.home.mindspring.com) with parameters: $M_{s}=$ $800 \times 10^{3} \mathrm{~A} / \mathrm{m}, A=13 \times 10^{-12} \mathrm{~J} / \mathrm{m}, \alpha=0.02, \beta=$ $0.08, j=3 \times 10^{12} \mathrm{~A} / \mathrm{m}^{2}, 5 \mathrm{~nm}$ cell size.

[26] J. Shibata et al., Phys. Rev. B 73, 020403(R) (2006). 